\title{
SUPPLEMENT TO “ON BAYESIAN “CENTRAL CLUSTERING”: APPLICATION TO LANDSCAPE CLASSIFICATION OF WESTERN GHATS
}

\author{
By SABYASACHI MUKHOPADHYAY, ${ }^{*}$, \\ SOURABH BHATTACHARYA ${ }^{\dagger, \S}$ AND KAJAL DiHIDAR ${ }^{\ddagger}, \S$ \\ Indian Statistical Institute ${ }^{\S}$
}

Throughout, we refer to the main manuscript as MBD.

S-1. Full conditionals with respect to the non-marginalized version of SB's model. Letting $\left\{\boldsymbol{\theta}_{1}^{*}, \ldots, \boldsymbol{\theta}_{k}^{*}\right\}$ denote the distinct components in $\boldsymbol{\Theta}_{M}$, let us define the configuration vector $\boldsymbol{C}=\left\{c_{1}, \ldots, c_{M}\right\}$, where $c_{j}=\ell$ if and only if $\boldsymbol{\theta}_{j}=$ $\boldsymbol{\theta}_{\ell}^{*}$. Then the random variables to be updated are $\left(\boldsymbol{Z}, \boldsymbol{C}, k, \boldsymbol{\theta}_{1}^{*}, \ldots, \boldsymbol{\theta}_{k}^{*}, \alpha\right)$. The respective full conditionals are provided below.

S-1.1. Full conditional distributions of the allocation variables. The full conditional distribution of the allocation variables $z_{i}(i=1, \ldots, n)$ given the rest is given by

$$
\left[z_{i}=j \mid \boldsymbol{Y}, \boldsymbol{\Theta}_{M}, \boldsymbol{Z}_{-i},\right] \propto \frac{\left|\boldsymbol{\Lambda}_{j}\right|^{\frac{1}{2}}}{(2 \pi)^{\frac{d}{2}}} \exp \left\{-\frac{1}{2}\left(\boldsymbol{y}_{i}-\boldsymbol{\mu}_{j}\right)^{\prime} \boldsymbol{\Lambda}_{j}\left(\boldsymbol{y}_{i}-\boldsymbol{\mu}_{j}\right)\right\} ; j=1, \ldots, M
$$

S-1.2. Full conditional distributions of the configuration indicators $C$. Let $k_{j}$ denote the number of distinct values in $\boldsymbol{\Theta}_{-j M}$, and let $\boldsymbol{\theta}_{\ell}^{j^{*}} ; \ell=1, \ldots, k_{j}$ denote the distinct values. Also suppose that $\boldsymbol{\theta}_{\ell}^{j^{*}}$ occurs $M_{\ell j}$ times.

Then the conditional distributions of $c_{j}$ are given, in the multivariate case, by

$$
\left[c_{j}=\ell \mid \boldsymbol{Y}, \boldsymbol{Z}, \boldsymbol{C}_{-j}, \boldsymbol{\Theta}_{M}\right] \propto\left\{\begin{array}{c}
q_{\ell j}^{*} \text { if } \ell=1, \ldots, k_{j} \\
q_{0 j} \text { if } \ell=k_{j}+1
\end{array}\right.
$$

* Sabyasachi Mukhopadhyay is a PhD student in Bayesian and Interdisciplinary Research Unit, Indian Statistical Institute

${ }^{\dagger}$ Sourabh Bhattacharya is an Assistant Professor in Bayesian and Interdisciplinary Research Unit, Indian Statistical Institute

${ }^{\ddagger}$ Kajal Dihidar is an Associate Scientist in Applied Statistics Unit, Indian Statistical Institute 
$q_{0 j}$ is given by

$$
\begin{aligned}
q_{0 j} & =\alpha\left(\frac{1}{n_{j} \psi+1}\right)^{\frac{d}{2}} \times\left(\frac{1}{\pi}\right)^{\frac{n_{j} d}{2}} \times \frac{|\boldsymbol{S}|^{\frac{s}{2}}}{\prod_{l=1}^{d} \Gamma\left(\frac{s+1-l}{2}\right)} \\
& \times \frac{\prod_{l=1}^{d} \Gamma\left(\frac{s+n_{j}+1-l}{2}\right)}{\left|\left\{\boldsymbol{S}+\frac{n_{j}\left(\overline{\boldsymbol{y}}_{j}-\boldsymbol{\mu}_{0}\right)\left(\overline{\boldsymbol{y}}_{j}-\boldsymbol{\mu}_{0}\right)^{\prime}}{n_{j} \psi+1}+\sum_{i: z_{i}=j}\left(\boldsymbol{y}_{i}-\overline{\boldsymbol{y}}_{j}\right)\left(\boldsymbol{y}_{i}-\overline{\boldsymbol{y}}_{j}\right)^{\prime}\right\}\right|^{\frac{s+n_{j}}{2}}}
\end{aligned}
$$

and

$q_{\ell j}^{*}=M_{\ell j} \frac{\left|\boldsymbol{\Lambda}_{\ell}^{j^{*}}\right|^{\frac{n_{j}}{2}}}{(2 \pi)^{\frac{n_{j} d}{2}}} \exp \left[-\frac{1}{2}\left\{n_{j}\left(\boldsymbol{\mu}_{\ell}^{j^{*}}-\overline{\boldsymbol{y}}_{j}\right)^{\prime} \boldsymbol{\Lambda}_{\ell}^{j^{*}}\left(\boldsymbol{\mu}_{\ell}^{j^{*}}-\overline{\boldsymbol{y}}_{j}\right)+\operatorname{tr} \boldsymbol{\Lambda}_{\ell}^{j^{*}} \sum_{i: z_{i}=j}\left(\boldsymbol{y}_{i}-\overline{\boldsymbol{y}}_{j}\right)\left(\boldsymbol{y}_{i}-\overline{\boldsymbol{y}}_{j}\right)^{\prime}\right\}\right]$

S-1.3. Full conditional distributions of the distinct values of $\Theta_{M}$. The conditional posterior distribution of $\boldsymbol{\theta}_{\ell}^{*}$ is given by

$$
\left[\boldsymbol{\theta}_{\ell}^{*} \mid \boldsymbol{Y}, \boldsymbol{Z}, \boldsymbol{C}\right] \sim \text { Wishart }_{d}\left(\boldsymbol{\Lambda}_{\ell}^{*}: \frac{s_{\ell}^{*}}{2}, \frac{\boldsymbol{S}_{\ell}^{*}}{2}\right) \times N_{d}\left(\boldsymbol{\mu}_{\ell}^{*}: \boldsymbol{\mu}_{0 \ell}^{*}, \psi_{\ell}^{*} \boldsymbol{\Lambda}_{\ell}^{*-1}\right)
$$

In the above, $n_{\ell}^{*}=\sum_{j: c_{j}=\ell} n_{j}, \overline{\boldsymbol{y}}_{\ell}^{*}=\sum_{j: c_{j}=\ell} n_{j} \overline{\boldsymbol{y}}_{j} / \sum_{j: c_{j}=\ell} n_{j}, s_{\ell}^{*}=n_{\ell}^{*}+s$, $\boldsymbol{\mu}_{0 \ell}^{*}=\left(\psi n_{\ell}^{*} \overline{\boldsymbol{y}}^{*}+\boldsymbol{\mu}_{0}\right) /\left(\psi n_{\ell}^{*}+1\right)$,

$$
\begin{aligned}
\boldsymbol{S}_{\ell}^{*} & =\boldsymbol{S}+\frac{n_{\ell}^{*}\left(\boldsymbol{\mu}_{0}-\overline{\boldsymbol{y}}_{\ell}^{*}\right)\left(\boldsymbol{\mu}_{0}-\overline{\boldsymbol{y}}_{\ell}^{*}\right)^{\prime}}{\psi n_{\ell}^{*}+1} \\
& +\sum_{j: c_{j}=\ell} n_{j}\left(\overline{\boldsymbol{y}}_{j}-\overline{\boldsymbol{y}}_{\ell}^{*}\right)\left(\overline{\boldsymbol{y}}_{j}-\overline{\boldsymbol{y}}_{\ell}^{*}\right)^{\prime} \\
& +\sum_{j: c_{j}=\ell} \sum_{i: z_{i}=j}\left(\boldsymbol{y}_{i}-\overline{\boldsymbol{y}}_{j}\right)\left(\boldsymbol{y}_{i}-\overline{\boldsymbol{y}}_{j}\right)^{\prime},
\end{aligned}
$$

and $\psi_{\ell}^{*}=\psi /\left(\psi n_{\ell}^{*}+1\right)$. It is to be noted that the $\boldsymbol{\theta}_{\ell}^{*}$ are conditionally independent.

The allocation variables $\boldsymbol{Z}$ can then be updated following (1) after transforming the configuration indicators and the distinct parameter values back to the original parameters.

S-1.4. Full conditional distribution of $\alpha$. Given the prior $\alpha \sim \operatorname{Gamma}\left(a_{\alpha}, b_{\alpha}\right)$, $k$ distinct components, and another continuous random variable $\eta$, the full conditional of $\alpha$ is

$$
\begin{aligned}
{\left[\alpha \mid \boldsymbol{Y}, \boldsymbol{Z}, \boldsymbol{\Theta}_{M}, k, \eta\right] } & \sim \pi_{\eta} \operatorname{Gamma}\left(a_{\alpha}+k, b_{\alpha}-\log (\eta)\right) \\
& +\left(1-\pi_{\eta}\right) \operatorname{Gamma}\left(a_{\alpha}+k-1, b_{\alpha}-\log (\eta)\right)
\end{aligned}
$$


where

$$
\frac{\pi_{\eta}}{1-\pi_{\eta}}=\frac{a_{\alpha}+k-1}{m\left(b_{\alpha}-\log (\eta)\right)}
$$

The full conditional distribution of $\eta$ given the rest is $\operatorname{Beta}(\alpha+1, M)$, that is, a Beta distribution with mean $(\alpha+1) /(\alpha+M+1)$.

\section{S-2. Full conditionals with respect to the marginalized version of SB's model.}

S-2.1. Full conditional distributions of the allocation variables $\boldsymbol{Z}$. Suppose that there are $k$ distinct components in $\boldsymbol{C}$. Also, in $n_{\ell}^{*}, \overline{\boldsymbol{y}}_{\ell}^{*}, s_{\ell}^{*}, \psi_{\ell}^{*}, \boldsymbol{\mu}_{0 \ell}^{*}, \boldsymbol{S}_{\ell}^{*}$, let $z_{i}$ be replaced with $j$. Then the full conditional probability of $z_{i}=j$ is given by

$$
\left[z_{i}=j \mid \boldsymbol{Y}, \boldsymbol{Z}_{-i}, \boldsymbol{C}\right] \propto \prod_{\ell=1}^{k}\left\{\frac{\prod_{t=1}^{d} 2^{\frac{d s_{\ell}^{*}}{2}} \psi_{\ell}^{* \frac{d}{2}} \Gamma\left(\frac{s_{\ell}^{*}+1-t}{2}\right)}{\left|S_{\ell}^{*}\right|^{\frac{s_{\ell}^{*}}{2}}}\right\}
$$

S-2.2. Full conditional distributions of the configuration indicators $C$. Let $k_{j}$ denote the number of distinct values in $C_{-j}$, and let $c_{\ell}^{j^{*}} ; \ell=1, \ldots, k_{j}$ denote the distinct values. Also suppose that $c_{\ell}^{j^{*}}$ occurs $M_{\ell j}$ times.

Let us define the following:

$$
\begin{aligned}
n_{\ell}^{*(j)}= & \sum_{r \neq j: c_{r}=\ell} n_{r} \\
\overline{\boldsymbol{y}}_{\ell}^{*(j)=} & \sum_{r \neq j: c_{r}=\ell} n_{r} \overline{\boldsymbol{y}}_{r} / n_{\ell}^{*(j)} \\
s_{\ell}^{*(j)}= & s+n_{\ell}^{*(j)} \\
\psi_{\ell}^{*(j)=} & \psi /\left(\psi n_{\ell}^{*(j)}+1\right) \\
\boldsymbol{\mu}_{0 \ell}^{*(j)}= & \left(\psi n_{\ell}^{*(j)} \overline{\boldsymbol{y}}_{\ell}^{*(j)}+\boldsymbol{\mu}_{0}\right) /\left(\psi n_{\ell}^{*(j)}+1\right) \\
\boldsymbol{S}_{\ell}^{*(j)=} & \boldsymbol{S}+\frac{n_{\ell}^{*(j)}}{\psi n_{\ell}^{*(j)}+1}\left(\boldsymbol{\mu}_{0}-\overline{\boldsymbol{y}}_{\ell}^{*(j)}\right)\left(\boldsymbol{\mu}_{0}-\overline{\boldsymbol{y}}_{\ell}^{*(j)}\right)^{\prime} \\
& +\sum_{r \neq j: c_{r}=\ell} \sum_{i: z_{i}=r}\left(\boldsymbol{y}_{i}-\overline{\boldsymbol{y}}_{\ell}^{*(j)}\right)\left(\boldsymbol{y}_{i}-\overline{\boldsymbol{y}}_{\ell}^{*(j)}\right)^{\prime}
\end{aligned}
$$

Then the full conditional distribution of $c_{j}$ is given by

$$
\left[c_{j}=\ell \mid \boldsymbol{Y}, \boldsymbol{Z}, \boldsymbol{C}_{-j}\right] \propto\left\{\begin{array}{c}
q_{\ell j}^{*} \text { if } \ell=1, \ldots, k_{j} \\
q_{0 j} \text { if } \ell=k_{j}+1
\end{array}\right.
$$


where $k$ is the normalizing constant, $q_{0 j}$ is given by

$$
\begin{aligned}
& q_{\ell j}^{*}=M_{\ell j}\left(\frac{1}{n_{j} \psi_{\ell}^{*(j)}+1}\right)^{\frac{d}{2}} \times\left(\frac{1}{\pi}\right)^{\frac{n_{j} d}{2}} \times \frac{\left|\boldsymbol{S}_{\ell}^{*(j)}\right|^{s_{\ell}^{*(j)}}}{\prod_{l=1}^{d} \Gamma\left(\frac{s_{\ell}^{*(j)}+1-l}{2}\right)} \\
& \times \frac{\prod_{l=1}^{d} \Gamma\left(\frac{s_{\ell}^{*(j)}+n_{j}+1-l}{2}\right)}{\left|\left\{\boldsymbol{S}_{\ell}^{*(j)}+\frac{n_{j}\left(\overline{\boldsymbol{y}}_{j}-\boldsymbol{\mu}_{0 \ell}^{*}(j)\right)\left(\overline{\boldsymbol{y}}_{j}-\boldsymbol{\mu}_{0 \ell}^{*}(j)\right)^{\prime}}{n_{j} \psi_{\ell}^{*(j)}+1}+\sum_{i: z_{i}=j}\left(\boldsymbol{y}_{i}-\overline{\boldsymbol{y}}_{j}\right)\left(\boldsymbol{y}_{i}-\overline{\boldsymbol{y}}_{j}\right)^{\prime}\right\}\right|^{\frac{s_{\ell}^{*(j)}+n_{j}}{2}}}
\end{aligned}
$$

and $q_{0 j}$ is given by (3).

The full conditional distribution of $\alpha$ remains same as in the non-marginalized model, described in Section S-1.4.

$\mathrm{S}$-3. The $K$-means algorithm is a special case of the clustering method based on SB's model. Recall that with $M=n$ and $z_{i}=i$ for $i=1, \ldots, n$, the mixture model of SB reduces to the model of EW. The latter will be used below to prove the remaining part of the result.

We first assume that $\boldsymbol{\Sigma}_{j}=\sigma^{2} \mathbf{I}$, for $j=1, \ldots, n$, and $\boldsymbol{G}_{0}$ is a uniform (improper) density over the entire parameter space. We further assume that $\sigma^{2}$ is known.

Then consider the configuration vector $C=\left\{c_{1}, \ldots, c_{n}\right\}$, where $c_{j}=\ell$ if and only if $\boldsymbol{\mu}_{j}=\boldsymbol{\mu}_{\ell}^{*} ; j, \ell=1, \ldots, n$, where, given $k(\leq n),\left\{\boldsymbol{\mu}_{1}^{*}, \ldots, \boldsymbol{\mu}_{k}^{*}\right\}$ denotes the set of $k$ distinct values in the complete set $\left\{\boldsymbol{\mu}_{1}, \ldots, \boldsymbol{\mu}_{n}\right\}$.

It then follows (see, for example, Müller, Erkanli and West (1996)) that the posterior distribution of $\left\{\boldsymbol{\mu}_{1}^{*}, \ldots, \boldsymbol{\mu}_{k}^{*}\right\}$ given data $\boldsymbol{Y}$, the configuration vector $\boldsymbol{C}$, and $k$, is given by

$$
\begin{aligned}
\pi\left(\boldsymbol{\mu}_{1}^{*}, \ldots, \boldsymbol{\mu}_{k}^{*} \mid \boldsymbol{Y}, \boldsymbol{C}, k\right) & \propto \prod_{j=1}^{k} \prod_{i: c_{i}=j} \exp \left\{-\frac{1}{2 \sigma^{2}}\left(\boldsymbol{y}_{i}-\boldsymbol{\mu}_{j}^{*}\right)^{\prime}\left(\boldsymbol{y}_{i}-\boldsymbol{\mu}_{j}^{*}\right)\right\} \\
& =\exp \left\{-\frac{1}{2 \sigma^{2}} \sum_{j=1}^{k} \sum_{i: c_{i}=j}\left(\boldsymbol{y}_{i}-\boldsymbol{\mu}_{j}^{*}\right)^{\prime}\left(\boldsymbol{y}_{i}-\boldsymbol{\mu}_{j}^{*}\right)\right\}
\end{aligned}
$$

Since the prior $G_{0}$ is completely non-informative, the conditional Bayes estimators of $\left\{\boldsymbol{\mu}_{1}^{*}, \ldots, \boldsymbol{\mu}_{k}^{*}\right\}$, given $\boldsymbol{Y}, \boldsymbol{C}$, and $k$ must coincide with the conditional maximum likelihood estimators of $\left\{\boldsymbol{\mu}_{1}^{*}, \ldots, \boldsymbol{\mu}_{k}^{*}\right\}$, which must be obtained by minimising the 
sum-of-squares term

$$
S S=\sum_{j=1}^{k} \sum_{i: c_{i}=j}\left(\boldsymbol{y}_{i}-\boldsymbol{\mu}_{j}^{*}\right)^{\prime}\left(\boldsymbol{y}_{i}-\boldsymbol{\mu}_{j}^{*}\right),
$$

the negative of the exponential of (12). But minimisation of (13) is exactly the exercise employed to implement the $K$-means algorithm, given the clusters $\left\{\boldsymbol{y}_{i}\right.$ : $\left.c_{i}=j\right\} ; j=1, \ldots, k$. Also note that the elements of the configuration vector $\boldsymbol{C}=\left(c_{1}, \ldots, c_{n}\right)^{\prime}$ are such that, $c_{i}=j$ with high probability when $\boldsymbol{y}_{i}$ is close to $\boldsymbol{\mu}_{j}^{*}$. This can be seen by noting that the conditional probability of $c_{i}=j$ in the set up described above is proportional to $\exp \left\{-\frac{1}{2 \sigma^{2}}\left(\boldsymbol{y}_{i}-\boldsymbol{\mu}_{j}^{*}\right)^{\prime}\left(\boldsymbol{y}_{i}-\boldsymbol{\mu}_{j}^{*}\right)\right\}$. Thus, the conditional probability of $c_{i}=j$ will be higher than those of $c_{i}=\ell$, for $\ell \neq j$ if $\boldsymbol{y}_{i}$ is closer to $\boldsymbol{\mu}_{j}^{*}$ than $\boldsymbol{\mu}_{\ell}^{*}$, for $\ell \neq j$. Recall that this is the same principle used by the $K$-means algorithm to allocate the data to different clusters, albeit the latter allocates deterministically based on minimum distance, rather then probabilistically.

S-4. Properties of the distance measure $\hat{d}$. In this section we show that the approximate distance $\hat{d}$ satisfies the first three properties of a metric in general and the triangular inequality at least in the case of independent clusterings.

Clearly from the definition of $\hat{d}$, for any two clusterings $I$ and $I I, d(I, I I) \geq 0$. Also, symmetry holds by construction, that is, $\hat{d}(I, I I)=\hat{d}(I I, I)$.

Moreover, if two clusterings $I$ and $I I$ are equivalent in the sense that any one of $I$ and $I I$ can be obtained from the other by just a renumbering of the clusters, then the two way table contains only one non-zero element in each row and column. As a result, $\hat{d}(I, I I)=0$. On the other hand, $\hat{d}(I, I I)=0$ implies that both $\tilde{d}(I, I I)$ and $\tilde{d}(I I, I)$ are zero. But this can not be the case unless each row and column of the two way table contains a single non-zero element. The latter implies that the two way table can be reduced to a diagonal matrix by simply renumbering one of the clusters, and hence $I$ and $I I$ are equivalent. So, $\hat{d}(I, I I)=0$ if and only if $I$ and $I I$ are equivalent.

For the triangular inequality let us consider independent clusterings, that is, the situation where the elements $n_{i j}$ of the two-way table corresponding to two clusterings satisfy

$$
\frac{n_{i j}}{n_{00}}=\left(\frac{n_{i 0}}{n_{00}}\right)\left(\frac{n_{0 j}}{n_{00}}\right)
$$

In (14) $n_{i 0}=\sum_{j} n_{i j}, n_{0 j}=\sum_{i} n_{i j}$, and $n_{00}=\sum_{i} \sum_{j} n_{i j}=\sum_{i} n_{i 0}=\sum_{j} n_{0 j}$.

Let us consider two clusterings denoted by $I$ and $I I I$, each consisting of $k$ clusters (if the number of clusters are different, denoted by $k_{1}$ and $k_{2}$ with $k_{1}>k_{2}$, 
we can still form a square matrix of size $k_{1} \times k_{1}$ with the remaining elements taken as zeroes). We denote the marginals corresponding to clusterings $I$ and $I I I$ by $n_{i 0}^{(I)}$ and $n_{0 j}^{(I I I)}$, respectively.

Now let $\max _{1 \leq i \leq k} n_{i 0}^{(I)}=n_{i_{1} 0}^{(I)}, \max _{1 \leq j \leq k} n_{0 j}^{(I I I)}=n_{0 j_{1}}^{(I I I)}$, and $n_{0 j_{1}}^{(I I I)} \geq n_{i_{1} 0}^{(I)}$, where $i_{1}, j_{1} \in\{1, \ldots, k\}$. Then, it easily follows that

$$
\hat{d}(I, I I I)=1-\frac{n_{i_{1} 0}^{(I)}}{n_{00}}
$$

Now consider a third clustering of the same data set, denoted by $I I$. Let the marginal totals corresponding to this clustering be denoted by $n_{0 j}^{(I I)}$, and suppose that $\max _{1 \leq j \leq k} n_{0 j}^{(I I)}=n_{0 j_{2}}^{(I I)}$, where, as before, $j_{2} \in\{1, \ldots, k\}$. Further assuming that $n_{i_{1} 0}^{(I)} \geq n_{0 j_{2}}^{(I I)}$ we obtain

$$
\hat{d}(I, I I)=1-\frac{n_{0 j_{2}}^{(I I)}}{n_{00}}
$$

On the other hand, if $n_{i_{1} 0}^{(I)}<n_{0 j_{2}}^{(I I)}$, then we would have obtained

$$
\hat{d}(I, I I)=1-\frac{n_{i_{1} 0}^{(I)}}{n_{00}}
$$

Since $\hat{d}(I I, I I I) \geq 0$, it clearly holds in the latter case that

$$
\hat{d}(I, I I I) \leq \hat{d}(I, I I)+\hat{d}(I I, I I I)
$$

In the case $n_{i_{1} 0}^{(I)} \geq n_{0 j_{2}}^{(I I)}$, we must have from (15) and (16) that $\hat{d}(I, I I) \geq$ $\hat{d}(I, I I I)$. This obviously implies, since $\hat{d}(I I, I I I) \geq 0$, that the triangular inequality (18) holds.

Hence, the triangular inequality is satisfied when $n_{0 j_{1}}^{(I I I)} \geq n_{i_{1} 0}^{(I)}$. However, if $n_{0 j_{1}}^{(I I I)}<n_{i_{1} 0}^{(I)}$, then $\hat{d}(I, I I I)=1-\frac{n_{0 j_{1}}^{(I I I)}}{n_{00}}$. But $\hat{d}(I I, I I I)$ is either $1-\frac{n_{j_{3} 0}^{(I I)}}{n_{00}}$ $\left(n_{j_{3} 0}^{(I I)}=\max _{1 \leq i \leq k} n_{i 0}^{(I I)} ; j_{3} \in\{1, \ldots, k\}\right)$ or $1-\frac{n_{0 j_{1}}^{(I I I)}}{n_{00}}$, depending, respectively, upon the inequalities $n_{0 j_{1}}^{(I I I)} \geq n_{j_{3} 0}^{(I I)}$ and $n_{0 j_{1}}^{(I I I)}<n_{j_{3} 0}^{(I I)}$. In either case, the triangular inequality (18) is satisfied.

\section{S-5. Investigation of the importance of the spatial structure of the superpix-} els. To test the significance of the spatial structure of the superpixels we incorporated the two-dimensional spatial locations within each of the 51,834 4-variate 
data points to obtain 6-variate observations in each case. Then, with this new data set, we implemented the $K$-means algorithm to obtain a new clustering that takes the spatial locations into account. It follows that the sum of squares required to be minimized for implementing the $K$-means algorithm involves the euclidean distance between the spatial locations. If the spatial aspect is important, then the new $K$-means clustering obtained from clustering the 6-dimensional data is expected to be quite different from the $K$-means clustering without the spatial information. In other words, since this difference is measured with our clustering metric, a large value of the metric would indicate that the spatial structure is important. This distance turned out to be 0.635 . In order to assess the statistical significance of this distance, we computed the distribution of our clustering metric using MCMC samples from our model. The distributions, after fixing the $K$-means clustering, the spatial $K$-means clustering, the conditional central clustering $\left(C^{(1)}\right)$ and the unconditional central clusterings $\left(C^{(412)}\right.$ and $\left.C^{(759)}\right)$ are also calculated. Differences between conditional and unconditional central clusterings are discussed in Section 5.8 of MBD. Figure S-1, displaying the distributions of our clustering metric obtained from the MCMC samples, shows that this distance is statistically insignificant. In other words, we do not gain any staistically significant information by incorporating the spatial structure of the superpixels. Hence, our informal statistical analysis agrees with the decision of NG to ignore the spatial aspects of the superpixels and also reduce computational burden as a result.

S-6. Sensitivity analysis. To check sensitivity of the results with respect to different prior assumptions, we conducted the following experiments.

In the first experiment, we set $M=30$ as before, but changed the priors of the other parameters; for example, we chose $\alpha \sim \operatorname{Gamma}(0.1,1)$, that is, the prior mean of $\alpha$ is now 0.1 instead of 1 , and the variance is decreased drastically from 10 to 0.1 . Noting that $\alpha$ is the parameter that controls the distribution of the unknown number of components, the purpose of taking a much stronger prior on $\alpha$, which is also centered around 0.1 , a value drastically smaller than in the case of the previous prior, is to test whether or not the data contains enough information to wash away the effect of even this strong prior, centered around an entirely different value, to yield a posterior distribution of the unknown number of components similar to that corresponding to the original (and weaker) prior. Apart from changing the prior of $\alpha$, we also changed $\boldsymbol{\mu}_{0}$ to $\boldsymbol{\mu}_{1}=\boldsymbol{\mu}_{0}+a \mathbf{1}$ and $\boldsymbol{S}$ to $\boldsymbol{S}_{1}$ where the offdiagonal elements of $\boldsymbol{S}_{1}$ are the same as those of $\boldsymbol{S}$ but the diagonal elements are $b$ times those of $\boldsymbol{S}$. Here $a$ and $b(>0)$ are arbitrary values, $\mathbf{1}$ is the vector with each component 1, and $\boldsymbol{I}$ is the identity matrix. Here we report the results for $a=100$ and $b=0.01$. These choices indicate that the marginal prior distribution of $\boldsymbol{\mu}_{j}$ is highly concentrated around $\boldsymbol{\mu}_{1}$, which is very different from $\boldsymbol{\mu}_{0}$. Once again, 


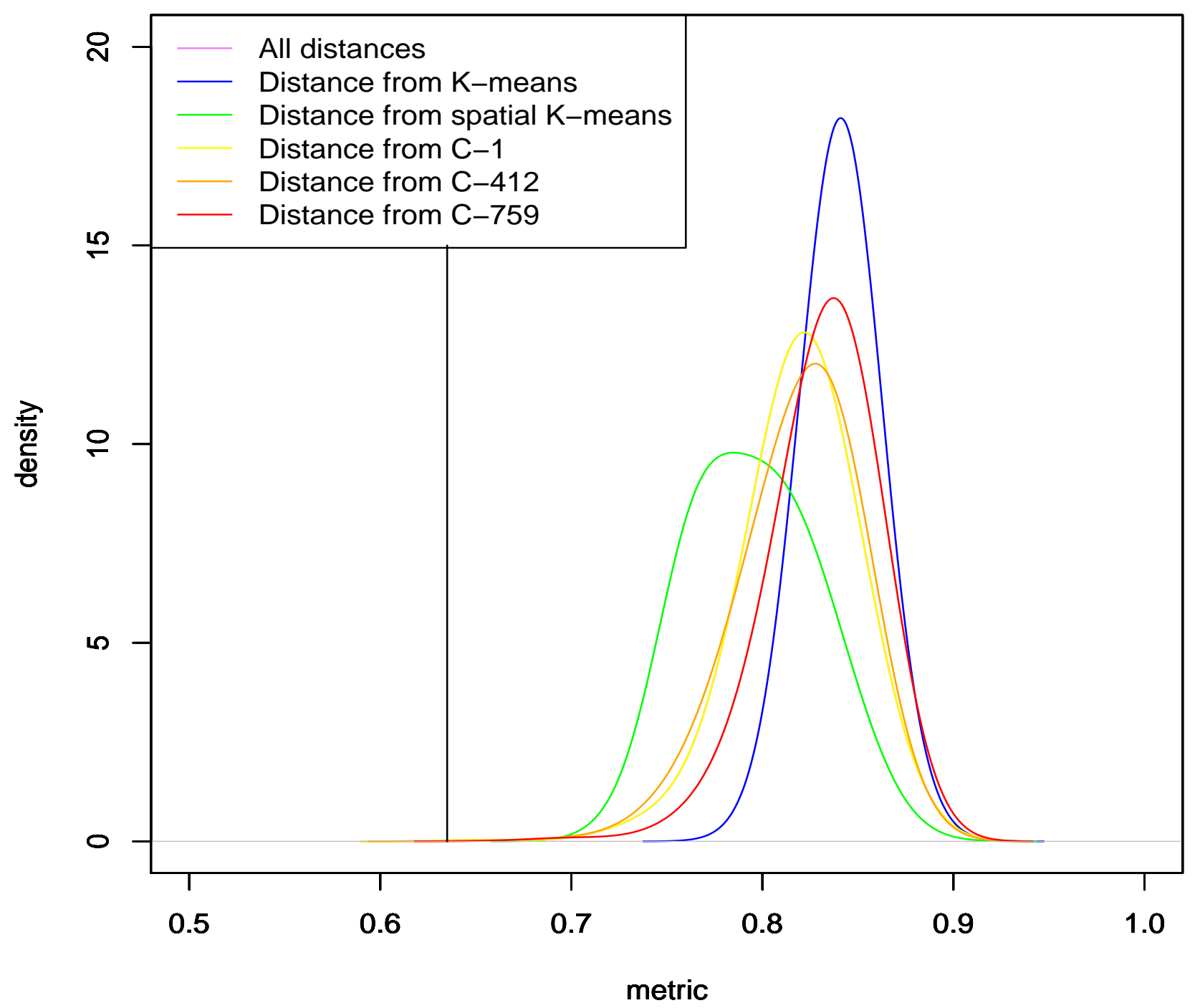

FIG S-1. Distributions of the clustering metric $\hat{d}$. The vertical line denotes the distance between $K$-means clusterings obtained with and without spatial structure of the superpixels. 
the purpose of taking such a strong prior is to test if the data contains enough information to ensure robustness of posterior inference in this challenging situation. The number of components having positive posterior mass are $\{6, \ldots, 18\}$, with respective posterior probabilities $\{0.00015,0.00520,0.03010,0.10780,0.20520$, $0.25085,0.20820,0.12200,0.04990,0.01600,0.00395,0.00060,0.00005\}$. The distribution is almost the same as that corresponding to the previous prior set up (see Section 5.4 of MBD), indicating robustness with respect to prior choice.

In the second experiment we used the original prior structure and values of the parameters and the hyperparameters but took $M=60$. Now, however, the posterior distribution of the number of clusters changed, with $\{11, \ldots, 27\}$ getting positive posterior probabilities given by $\{0.00005,0.00020,0.00140,0.00545,0.02395$, $0.06140,0.11995,0.17195,0.19475,0.18190,0.11990,0.06865,0.03225,0.01310$, $0.00380,0.00100,0.00030\}$. Now 19 components, and not 11 components gets the maximum probability. Indeed, greater number of components are more probable with $M=60$, as compared to that with $M=30$, which is not surprising since a priori, the expected number of components, approximately given by $\alpha \log \left(1+\frac{M}{\alpha}\right)$, is higher in the former model. We remark, however, that if $M$ were very large for both the cases, then it follows from the expression of the (approximate) prior expectation of the number of components that the posteriors of the number of components would be very similar for both the models for the respective different, albeit, very large values of $M$.

A further experiment, with $M=60$ but with the aforementioned changed values of the hyperparameters, yielded the following posterior distribution of the number of components which is very similar to the case with the original hyperparameter values but with $M=60$ : the number of components having positive posterior mass are $\{11, \ldots, 29\}$, with respective posterior probabilities $\{0.00005,0.00000$, $0.00070,0.00590,0.02360,0.06340,0.12030,0.17150,0.19645,0.17685,0.12525$, $0.06635,0.03205,0.01215,0.00420,0.00080,0.00030,0.00010,0.00005\}$.

Figure S-2 displays the distributions of our clustering metric $\hat{d}$ with respect to the above three experiments plus with respect to the original analysis with $M=30$. Note that the distributions of $\hat{d}$ corresponding to the two experiments conditional on $M=30$ are similar; those conditional on $M=60$ are similar as well. But given $M=60, \hat{d}$ takes on values higher than those given $M=30$. This is easily explained by first recalling that greater numbers of components are more probable with $M=60$, and then noting that this implies that the maximum element in each row and column, associated with the calculation of $\hat{d}$ (see (14) of MBD), must decrease since the total number of units is fixed. This decreases the sum of the maxima, which, in turn, increases the value of the clustering metric.

These experiments indicate that the posterior, although very robust with respect to the choices of the other priors, is sensitive to the choice of $M$. Thus $M$ must be 


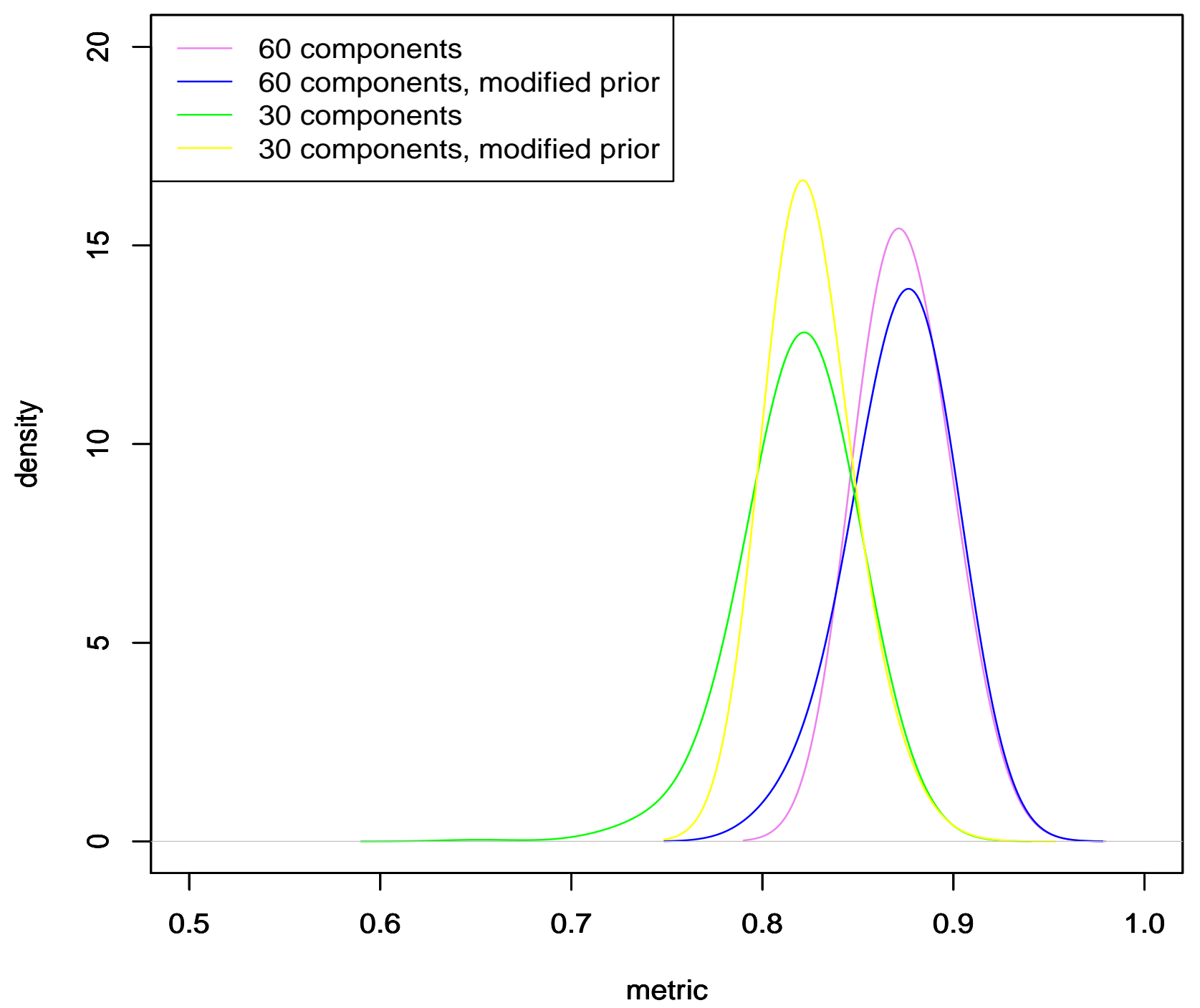

FIG S-2. Distributions of the clustering metric $\hat{d}$ under different $M=30$ and $M=60$, each with two different sets of priors 
carefully selected; a relatively "safe choice", which we adopt in this paper, may be the one that yields results similar in some sense to established scientific results.

S-7. Discussion of computational efficiency of SB's model compared to EW's model. High computational complexity is associated with the calculation of individual multinomial (full conditional) probabilities of each configuration indicator, under both EW's (see, for example, Müller, Erkanli and West (1996)) and SB's model (see Section S-1.2). But in SB's model the expected number of such computationally demanding multinomial probabilities is much less than that of EW. The reason is that the configuration indicators can take as many values as the number of distinct components, and it follows from Antoniak (1974) that a priori, in SB's model the expected number of distinct components is approximately $\alpha \log \left(1+\frac{M}{\alpha}\right)$, a bounded quantity, while that associated with EW's model is $\alpha \log \left(1+\frac{n}{\alpha}\right)$, which increases indefinitely with the sample size $n$. Hence, the expected computational cost for simulating a single configuration indicator is much less for SB's model than for EW's model. Moreover, since $n$ configuration indicators must be simulated for EW's model, the high computational cost for simulating each such indicator is multiplied $n$ times. On the other hand, in SB's case, the comparatively much low computational cost is multiplied only $M$ times thanks to only $M$ configuration indicators, where $M$ is negligibly small compared to $n$, the size of the massive data. Apart from simulating $C$, simulation of $\boldsymbol{Z}$ is also needed in SB's model, but in this case the computational cost is extremely small, with simulation of $z_{i}$ requiring evaluation of only a multivariate normal density at a data point only $M$ number of times. Notably, in the step for simulating $z_{i}$, the mean vector and the covariance matrix of this multivariate normal density (see (1) of Section S-1.1) are given, and so, unlike in the case of $c_{j}$, no calculation is associated with these parameters. Indeed, the total computational cost of simulating $Z$ and $C$ is negligible compared to that of simulating $C$ in EW's model. In the non-marginalized versions of the models, simulation of the distinct parameters are needed. A smaller expected number of distinct components in SB's model makes the computational cost smaller compared to that corresponding to EW's model. In other words, the overall computational cost associated with SB's model is far less than that associated with EW's model.

In the marginalized versions of $\mathrm{EW}$ and $\mathrm{SB}$, computations of the marginal probabilities associated with $C$ (and $Z$ in the case of SB) are much more involved compared to those associated with the non-marginalized versions. Hence, the computational costs of the implementations increase manifold, rendering both the versions useless for the massive Western Ghats data. But compared to EW, SB's model exhibited greater computational efficiency even in this case due to the smaller (and bounded) expected number of multinomial probabilities of $\boldsymbol{C}$, fixed $(M)$ number 
of multinomial probabilities of $\boldsymbol{Z}$, and lesser number of configuration indicators.

S-8. MCMC convergence diagnostics. It is important to provide some assessment of the convergence of our MCMC algorithm. To proceed, we divide our MCMC sample of size $N$ into, say, $m$ equal parts, each part having the same size $N / m$, assuming $N$ is divisible by $m$. Given each such subsample of size $m$, we compute a central clustering and the corresponding approximate $95 \%$ credible region of the clusterings. If the $m$ credible regions thus obtained are close to each other, one can safely infer that the $m$ samples arose from the same stationary distribution. Now there arises the question as to how can one ascertain if two 95\% credible regions, obtained from two separate samples, are close enough? We propose the following idea.

Let $\left(C R_{\epsilon_{1}}, \epsilon_{1}\right)$ and $\left(C R_{\epsilon_{2}}, \epsilon_{2}\right)$ denote the $95 \%$ credible regions and the corresponding radii obtained from any two subsamples. Suppose that $\eta_{1}>0$ is the least positive value such that $C R_{\epsilon_{1}+\eta_{1}} \supseteq C R_{\epsilon_{2}}$, and also suppose that $\eta_{2}>0$ is the least positive value such that $C R_{\epsilon_{2}+\eta_{2}} \supseteq C R_{\epsilon_{1}}$. Then, if both the increments $\eta_{1}, \eta_{2}$ are sufficiently small, the $95 \%$ credible regions $C R_{\epsilon_{1}}$ and $C R_{\epsilon_{2}}$ can be said to be "close".

Observe that it is not necessary for convergence diagnostics to consider clustering of data; clustering of $\boldsymbol{\Theta}_{M}$ is also appropriate and is computationally less expensive when $M<<n$, as in our Western Ghats example. In our implementation of the Gibbs sampler associated with SB's model, all the increments turned out to be less than or equal to $2 \times 10^{-6}$, irrespective of the data clusterings or $\Theta_{M}$-clusteirngs. This strongly indicates convergence of our Gibbs sampler.

We remark that a related MCMC convergence diagnostic test can be found in Robert and Casella (2004), who propose to test equality of distributions corresponding to samples obtained by splitting up the entire MCMC sample by the Kolmogorov-Smirnov test. Such a test, however, is rendered ineffective in our setup, where distributions of clusterings, rather than real-valued parameters, are of interest.

\section{References.}

AntoniaK, C. E. (1974). Mixtures of Dirichlet Processes With Applications to Nonparametric Problems. The Annals of Statistics 2 1152-1174.

MüLler, P., ERKANLI, A. and WeSt, M. (1996). Bayesian curve fitting using multivariate normal mixtures. Biometrika 83 67-79.

Robert, C. P. and Casella, G. (2004). Monte Carlo Statistical Methods. Springer-Verlag, New York.

BAYESIAN AND INTERDISCIPLINARY RESEARCH UNIT BAYESIAN AND INTERDISCIPLINARY RESEARCH UNIT INDIAN STATISTICAL INSTITUTE

203, B. T. ROAD, KOLKATA 700108 INDIAN STATISTICAL INSTITUTE

E-MAIL: sabstat123@gmail.com

E-MAIL: bhsourabh@gmail.com 
APPLied STATISTICS UNit

INDIAN STATISTICAL INSTITUTE

203, B. T. ROAD, KolKata 700108

E-MAIL: dkajal@isical.ac.in 